\title{
Chapter 3 \\ Negotiating Privacy Concerns \\ and Social Capital Needs in a Social \\ Media Environment
}

\author{
Nicole B. Ellison, Jessica Vitak, Charles Steinfield, Rebecca Gray, \\ and Cliff Lampe
}

\subsection{Introduction}

Social network sites (SNSs) are becoming an increasingly popular resource for both students and adults, who use them to connect with and maintain relationships with a variety of ties. For many, the primary function of these sites is to consume and distribute personal content about the self. Privacy concerns around sharing information in a public or semi-public space are amplified by SNSs' structural characteristics, which may obfuscate the true audience of these disclosures due to their technical properties (e.g., persistence, searchability) and dynamics of use (e.g., invisible audiences, context collapse) (boyd 2008b). Early work on the topic focused on the privacy pitfalls of Facebook and other SNSs (e.g., Acquisti and Gross 2006; Barnes 2006; Gross and Acquisti 2005) and argued that individuals were (perhaps inadvertently) disclosing information that might be inappropriate for some audiences, such as future employers, or that might enable identity theft or other negative outcomes.

The focus of this early work on negative outcomes of use, in the absence of research that considered motivations for use, presented a confusing portrait of the Facebook user. Our initial research exploring the "benefits of Facebook Friends" (Ellison et al. 2007) was inspired by the discrepancy between high usage patterns and a focus on negative outcomes. Our research has employed the social capital framework as a way of exploring the positive outcomes of SNS use. A stream of research by the authors has explored social capital outcomes of Facebook use (Ellison et al. 2007, 2010, 2011; Steinfield et al. 2009). The social capital approach has been replicated in other contexts, such as Valenzuela et al.'s (2009) study of Facebook use and civic engagement.

N.B. Ellison $(\triangle) \cdot J$. Vitak $\bullet$ C. Steinfield $\bullet$ R. Gray $\bullet$ C. Lampe

Michigan State University, East Lansing, MI, USA

e-mail: nellison@msu.edu 
One question not yet addressed by scholarship in this area is the relationship between privacy and social capital outcomes. Our conception of privacy speaks to the ability of individuals to control when, to what extent, and how information about the self is communicated to others (see Westin 1967; see also Chap. 2 of this volume for a further elaboration on theories of privacy by Margulis). In many cases, disclosing information about the self is necessary in order to reap the benefits from these technological tools. After all, members of one's social network cannot suggest a new job possibility if they do not know s/he is looking, nor can they offer social support if they do not know it is needed. By lowering the barriers to communicating with a wider network of weak ties (Donath and boyd 2004; Ellison et al. 2007), SNSs enable individuals to broadcast requests for support or information. Self-disclosure is also a means by which individuals learn about and develop relationships with one another (Berger and Calabrese 1975); however, this process entails revealing information about the self that one might not want to share with a wider audience.

This chapter will consider how SNS users balance the desire to share personal information (and thus potentially accrue the social capital benefits associated with disclosure) and the need to control these disclosures (by minimizing the risks associated with sharing private information). We describe three strategies by which users can control the audience for their disclosures on SNSs: Friending behaviors, managing audiences via privacy settings, and disclosures on the site. Below we briefly discuss social capital, privacy, and information disclosure on SNSs before presenting some preliminary findings about SNS privacy behaviors and social capital.

\subsection{Literature Review: Overview of Social Capital}

The concept of social capital has received considerable attention across numerous disciplines over the past three decades (Adler and Kwon 2002). Social capital broadly refers to the accumulated resources derived from the relationships among people within a specific social context or network (Bourdieu 2001; Coleman 1988; Lin 2001; Portes 1998; Putnam 2000). Some have expressed concern that the concept lacks theoretical and operational rigor - for example, Portes (1998) notes that conceptualizations of social capital can alternatively refer to the mechanisms that generate it (the relationships between people) or its outcomes (the resources one may obtain from these relationships). We emphasize social capital as an outcome that stems from relationships among people. Hence, being embedded in a network of relationships is a necessary precursor of social capital, but in and of itself is not synonymous with social capital.

Putnam (2000) distinguishes between two forms of social capital: one emanating from weak ties that he calls bridging social capital, and a second that is derived from strong or intimate ties like family relations, called bonding social capital. Bridging social capital is best understood in relation to groundbreaking work by 
Granovetter (1973), who observed that weak ties tend to be outside of one's dense local network and, by virtue of these ties having links to new people, help promote the diffusion of non-redundant information. One's strong ties, however, are likely to be connected to each other, suggesting that much of the information flowing through a close-knit network of relationships is redundant. Such strong ties are a source of bonding social capital and are associated with trust, reciprocity, emotional support, and tangible resource provision (Putnam 2000).

More recently, researchers have examined how Internet use influences people's abilities to form and maintain social capital, given that it provides many new ways to interact with a wide variety of others ranging from close contacts to relative strangers (Resnick 2001; Wellman and Gulia 1999; Williams 2006). Ellison et al. (2010) summarize this body of literature by grouping the findings into three basic categories: (1) those that find that Internet use enables people to generate new social capital (e.g., Hampton and Wellman 2003; Rheingold 1993), (2) those that find that Internet use diminishes people's stock of social capital (e.g., Kraut et al. 1998; Nie 2001), and (3) those that find that Internet use reinforces people's offline relationships and supplements social capital development (e.g., Quan Haase and Wellman 2004; Uslaner 2000).

We view social capital as a particularly relevant outcome to consider when examining use of SNSs, given that many of the core features of such sites are explicitly designed to facilitate the formation and maintenance of connections among people - connections that are sustained through communication about the self. Our own and others' research in the past half decade provides strong empirical support for the hypothesis that greater use of SNSs is associated with different types of social capital benefits (Burke et al. 2010; Ellison et al. 2007, 2011; Steinfield et al. 2008, 2009). Ellison et al. (2007) found that, even after controlling for a range of demographic attributes, general Internet use, and psychological well-being, the more intensely students used Facebook, the greater their reported bridging, bonding, and maintained social capital. Steinfield et al. (2008) investigated bridging social capital and Facebook use longitudinally, finding evidence for a causal effect of SNS use on levels of bridging social capital. Research in organizational settings also suggests a positive association between SNS use and both bridging and bonding social capital (Steinfield et al. 2009). Ellison et al. (2011) extended this work, finding that not all usage of Facebook resulted in social capital growth. Rather, students who reported greater use of Facebook in a social informationseeking capacity - specifically to learn more about people with whom they had some form of offline connection - had higher levels of social capital. Finally, using a sample of adult US Facebook users, Burke et al. (2010) found that more active users of Facebook (i.e., those who engaged in directed communication) reported higher levels of bridging and bonding social capital.

While the general relationship between SNS use and social capital has been established in a number of studies, to date no academic work has considered how privacy relates to social capital in the SNS context. We take this question up in the next section. 


\subsection{Privacy and SNSs: An Overview}

In defining SNSs, boyd and Ellison (2008) assert that SNSs contain three components that distinguish them from other online sites: (1) a user-constructed public or semi-public profile, (2) a set of connections to other users within the system, and (3) the ability to view one's own list of connections, as well the connections made by others in the system. Indeed, these public displays of connections are a defining feature of SNSs, differentiating them from most other forms of social media (Donath and boyd 2004). Decisions about whom to connect with on SNSs are a key component of users' ability to control their personal information. Similarly, users can control access to personal information through their disclosure behaviors - the kinds of information they include on their profile or share via status updates. A third critical area, and the subject of much of the literature, revolves around privacy settings.

Previous research examining privacy on SNSs is in disagreement over how privacy settings, Friending behaviors, and disclosures interact. For example, while Acquisti and Gross (2006) found little relationship between privacy concerns and certain types of disclosures, more recent studies have found that a high level of privacy concerns leads to fewer disclosures on SNSs (Krasnova et al. 2010; Stutzman et al. 2011). The relationship between these variables is further complicated by the presence of multiple audiences (e.g., high school friends, family, coworkers) within a single space (boyd 2008b), and users may employ a variety of strategies to mitigate risks associated with disclosures made to unintended audiences, such as using pseudonyms or employing advanced privacy controls.

In an online realm where individuals may benefit from sharing personal information, control over the audience for this information is critical. Privacy on SNSs is a multi-faceted issue, requiring attention on the user's part, both to protect information from third-party data collection and to manage personal impressions across a variety of contexts and relationships. The relationship between privacy concerns and privacy behaviors is complex. Facebook users generally believe that others in their network are more at risk than they are in regards to negative privacyrelated outcomes (Debatin et al. 2009). Past research on privacy and SNSs has explored the relationship between privacy concerns and actual behavior on SNSs, privacy "violations" that have left SNS users feeling vulnerable, and the distinction between social privacy and institutional privacy. For example, Acquisti and Gross (2006) found that one's privacy concerns were a weak predictor of SNS use, and that among those who had joined an SNS, there were no differences in the likelihood to make disclosures such as one's birthday, mobile number, or address between those who reported a high level of privacy concerns and those who reported low-level concern. Tufekci (2008) found similar results regarding the relationship between privacy concerns and disclosures through an SNS, but also found that students employed audience management strategies such as using a nickname or adjusting profile visibility. 
Perhaps the greatest focus of SNS privacy literature has been user awareness of settings and visibility to others using the site. It was not uncommon for early SNS researchers to find Facebook users relatively unaware of the activity, accessibility, and extent of their social networks despite reporting privacy concerns (Acquisti and Gross 2006; Strater and Richter 2007). In their study of Facebook users' attention to and use of privacy controls, Strater and Richter (2007) found that participants often experienced difficulty navigating the privacy settings of the Facebook interface during interviews, while Barnes (2006) observed that teenage SNS users appeared unaware or ignorant of the public nature of the content they shared through the sites. In more recent work, however, Stutzman and Kramer-Duffield (2010) found that $83 \%$ of respondents indicated using any Facebook privacy settings, while $58 \%$ of respondents indicated they had made their Facebook profile Friends-only.

Structural changes to Facebook have elicited public discussions about privacy issues and SNS use. In September 2006, Facebook introduced the News Feed, which aggregated the activities of a user's Friends and presented them in a reverse chronological order stream on the user's homepage. This meant that behaviors that were previously visible only by visiting one's profile, such as adding a Friend or joining a group, were highlighted in the News Feeds of one's Facebook Friends. The new visibility of Facebook activities inevitably left some Facebook users feeling as though they needed to monitor actions they formerly performed without hesitation; as boyd (2008a) wrote, "With Facebook, participants have to consider how others might interpret their actions, knowing that any action will be broadcast to everyone with whom they consented to digital Friendship" (p. 16).

\subsection{Identity and Information Disclosure in SNSs}

While both research and popular narratives point to numerous privacy concerns associated with using SNSs (Acquisti and Gross 2006; Lenhart and Madden 2007), information disclosures on SNSs - through one's profile information, interactions with other users, and the public display of one's connections - seem to be a necessary component of accruing benefits from one's network. As noted by Ellison et al. (2010), the information provided in SNS profiles (e.g., contact information, background data, personal characteristics) can lower the barriers to initial interaction and facilitate formation of common ground. Studies indicate that trust and willingness to share information were higher on Facebook, which requires users to provide their real name, than on MySpace, which does not have such a requirement (Dwyer et al. 2007). Furthermore, research by Mazer et al. (2009) found that perceptions of credibility on an SNS increased with greater information disclosure.

In reviewing the extant literature on self-presentation through SNS profiles, Ellison et al. (2010) conclude that access to personal identity information supports the relationship-formation process. Moving beyond purely "social" SNSs such as Facebook, DiMicco et al. (2009) provide support for this argument through a study of workplace SNS use, finding that employees use profile information to engage in 
"people sensemaking," which the authors describe as "the process a person goes through to get a general understanding or gist of who someone is" (p. 1). In other words, information gathered from a user's profile may aid in establishing common ground, which, in turn, may facilitate communication and coordination processes (Clark and Brennan 1991; Olson and Olson 2000). Research suggests that profile information in Facebook may help users find common ground with one another (DiMicco and Millen 2007; Dwyer et al. 2007; Lampe et al. 2007) and support relationships. For example, Lampe et al. (2007) grouped profile elements into three distinct categories - referent, interest, and contact information - and found that the more information users completed in each of these profile categories, the greater the size of their network, thus suggesting that disclosures within the profile aid in relationship formation. Another category of information included in a user's profile - the display of friend networks - may also serve to establish common ground and encourage more honest self-disclosures (see e.g., Donath and boyd 2004).

In addition to the role that the public display of connections may play in vetting a user's identity, users may consider their audience prior to making disclosures through an SNS. Recent work by Marwick and boyd (2011) and Hogan (2010) has begun to consider how individuals navigate audiences through social media, focusing on the concept of context collapse, or the idea that sites such as Facebook flatten audiences and make it challenging to employ different self-presentational strategies for different groups and individuals on the site. Privacy settings may help segregate audiences, but as Hogan suggests, users may simply take a lowest common denominator approach and only make disclosures that are appropriate for all members of their network. As with other privacy-based concerns, SNS users must balance concerns about their content being viewable by a variety of audiences with their desire to receive benefits from interactions on the site.

Recent research takes a more granular approach to exploring how user activity influences overall outcomes on SNSs. This work suggests that in order to reap benefits from use, dynamic disclosure beyond entering information into profile fields is needed. Burke et al. (2010) obtained both server-level and survey data from a large $(\mathrm{N}=1,193)$ sample of Facebook users and found that users who were actively engaged with Facebook had higher levels of social capital and other measures of well-being. They identified a "consumption" pattern of use (similar to lurkers in other contexts) comprised of users who clicked on Friends' profiles but did not contribute content themselves. This type of use was not associated with greater social capital levels and, in fact, was associated with increased loneliness. On the other hand, users who posted often and engaged in directed communication with Friends reported higher bonding social capital. Similarly, Kim and Lee (2011) find that honest self-presentation contributes indirectly to subjective well-being and is mediated by perceived social support. They write, "Facebook friends are more likely to provide support when they know that the user is in need for support; only when such need is properly communicated through self-disclosure facilitated by honest self-presentation are users likely to receive support from Facebook friends (p. 362)." Other work has examined bloggers' self-disclosure behavior, finding a 
similar relationship between self-reported disclosure and social capital measures (Ko and Kuo 2009).

In summary, research suggests that the provision of identity information and other disclosures on SNSs are key to extracting relational benefits from their use, but the large, diverse networks supported by SNSs can complicate these disclosures through context collapse and other considerations.

\subsection{A Preliminary Investigation of Privacy and Social Capital}

The relationship between privacy and social capital is complex. At the most basic level, it seems reasonable to assume that in order to accrue social capital benefits from one's social network, an individual must disclose information about the self, which may entail privacy concessions. For example, a Facebook user who only accepts friend requests from close offline friends may lack access to the bridging benefits associated with having a diverse network of weaker acquaintances. Likewise, Facebook users who do not actively engage in direct interaction through the site but instead spend their time reading content by others should be less likely to reap bonding benefits, such as emotional support, through the site.

We conducted two studies in 2010 that explored factors related to privacy and social capital, including users' privacy settings, Friending habits, disclosures on the site, and perceptions of social capital. The first was a survey of undergraduates at Michigan State University, while the second included interviews with a national sample of adult Facebook users. Below we discuss privacy-related findings of both studies.

\subsubsection{Quantitative Analysis of Undergraduates}

Each year that we collect data from undergraduates on their use of Facebook, we include a few items probing privacy and social capital variables. In our most recent dataset, collected in March and April of 2010 ( $\mathrm{N}=299)$, we asked questions about their privacy settings, the types of disclosures they make on the site, and their Friending behaviors (such as the number of Facebook Friends and the number of "actual" friends in their Facebook network), as well as the bonding and bridging social capital measures used in previous research (see Ellison et al. 2007).

When looking specifically at possible privacy-enhancing behaviors, we asked about two basic strategies: changing privacy settings from the default and limiting specific content to individuals or groups within one's network. We believe this second item is of special interest when considering how users are managing audiences within an SNS; by taking a more granular approach to restricting access and distributing content, users may be more willing to make greater disclosures through the site, which in turn, could lead to greater social capital gains. We found 
that a majority of participants (78\%) reported engaging in this strategy of restricting access to content to specific Friends. To probe further into the relationship between this behavior and our other variables of interest, we ran a series of independent sample t-tests, using the advanced privacy settings measure as the grouping variable. Significant differences emerged between those who reported using this feature and those who had not for a number of variables. First, when looking at our social capital measures, we found that participants who employed these privacy settings reported higher perceived bonding and bridging social capital. Furthermore, this group of participants reported having more Facebook Friends as well as more "actual" friends within their Facebook network. See Table 3.1 for means, standard deviations, and t-scores.

Next, we focused on two Friending behaviors: the total number of Facebook Friends a user has connected with through Facebook, as well as their perceptions regarding how many Facebook Friends they consider to be "actual" friends. As these were both continuous variables, we created a dichotomous variable for each, encompassing the lowest and highest quartiles of responses. Independent sample t-tests revealed that, similar to our previous analysis, there were significant differences in participants' reported social capital, such that participants reporting the most Facebook Friends and actual (Facebook) friends reported greater perceived bonding and bridging social capital than those reporting the fewest number of Facebook and actual friends. See Table 3.2 for details.

Table 3.1 Results from independent sample t-tests for employing advanced privacy settings

\begin{tabular}{lllll}
\hline & \multicolumn{3}{c}{ Advanced privacy settings } \\
\cline { 2 - 5 } & Have not used this feature & Have used this feature \\
\hline $\begin{array}{c}\text { Bridging SC, } \mathrm{t}(368)=-3.64, \\
\mathrm{p}<0.001\end{array}$ & $\mathrm{M}=3.60$ & S.D. $=0.67$ & $\mathrm{M}=3.90$ & S.D. $=0.69$ \\
$\begin{array}{c}\text { Bonding SC, } \mathrm{t}(114)=-2.324, \\
\mathrm{p}=0.022\end{array}$ & $\mathrm{M}=3.51$ & S.D. $=1.03$ & $\mathrm{M}=3.79$ & S.D. $=0.80$ \\
$\begin{array}{c}\text { Facebook Friends, } \\
\mathrm{t}(174)=-4.08, \mathrm{p}<0.001\end{array}$ & $\mathrm{M}=343.17$ & S.D. $=223.26$ & $\mathrm{M}=462.40$ & S.D. $=284.93$ \\
$\begin{array}{c}\text { Actual" friends, } \\
\mathrm{t}(174)=-3.12, \mathrm{p}=0.002\end{array}$ & $\mathrm{M}=161.97$ & S.D. $=151.57$ & $\mathrm{M}=229.25$ & S.D. $=217.53$ \\
\hline
\end{tabular}

Table 3.2 Results from independent sample t-tests for Friending behaviors

\begin{tabular}{lllll}
\hline & \multicolumn{4}{c}{ Friending behaviors } \\
\cline { 2 - 5 } & Lowest quartile & \multicolumn{3}{c}{ Highest quartile } \\
\hline Facebook Friends & & & & \\
Bridging SC, $\mathrm{t}(189)=-6.53, \mathrm{p}<0.001$ & $\mathrm{M}=3.47$ & S.D. $=0.72$ & $\mathrm{M}=4.11$ & S.D. $=0.64$ \\
Bonding SC, $\mathrm{t}(178)=-5.32, \mathrm{p}<0.001$ & $\mathrm{M}=3.38$ & S.D. $=0.90$ & $\mathrm{M}=4.00$ & S.D. $=0.69$ \\
"Actual Friends" in Facebook network & & & & \\
Bridging SC, $\mathrm{t}(178)=-6.66, \mathrm{p}<0.001$ & $\mathrm{M}=3.54$ & S.D. $=0.81$ & $\mathrm{M}=4.20$ & S.D. $=0.56$ \\
Bonding SC, $\mathrm{t}(184)=-5.41, \mathrm{p}<0.001$ & $\mathrm{M}=3.46$ & S.D. $=0.95$ & $\mathrm{M}=4.10$ & S.D. $=0.69$ \\
\hline
\end{tabular}

For total Facebook Friends, lowest quartile is $<240$ Friends, highest quartile is $600+$ Friends. For actual friends, lowest quartile is $<61$ friends, highest quartile is $300+$ friends 
Finally, to address how disclosures fit into this framework, we ran analyses using a weak two-item original scale assessing participants' disclosure habits through the site (registering their agreement with the statements, "When I'm having a bad day, I post about it on Facebook" and "When I receive a good grade in class, I post about it on Facebook" on a five-point Likert-type scale ranging from Strongly Disagree to Strongly Agree). Participants indicated a below-midpoint level of agreement with these statements (scale $M=2.54$, $S . D .=1.13)$, suggesting that they are not using Facebook as a way to share certain types of personal information about themselves. Furthermore, we found no significant relationship between this variable and privacy settings, social capital, or Friending behaviors but suspect this is due to the weakness of this measure. We expect that a better measure of disclosures, such as that employed in Burke et al. (2010), or one that captures more interaction-based disclosures happening outside the "status update" context, would be more likely to produce insight into social capital and privacy behavior dynamics.

Overall, we believe this initial analysis supports our conceptualization of multiple possible privacy behaviors and their potential relationship to social capital, although more granular measures and multivariate analyses are needed to flesh out these dynamics more fully. For example, the positive relationship between use of advanced privacy settings and the number of Friends (both total and actual) may reflect a strategy by which users with larger Friend counts (which are more likely to include those from different spheres) need to place these friends into groups, or the fact that those who feel comfortable creating lists also feel more comfortable accepting different types of people as Friends. The positive relationship between participants' use of the advanced privacy settings and both bridging and bonding social capital suggests that tools for managing audiences within an SNS may aid users' efforts to maximize rewards derived from interactions with network members, perhaps because users who are able to direct their disclosures to a subset of Friends may actually disclose more deeply and honestly. This interpretation contains face validity, especially in light of the positive relationship between both forms of social capital and participants' reported Facebook and actual friends on the site (potentially reflecting wider, more diverse networks and greater access to close friends).

While our measure of disclosures was extremely limited, it could be that users employ privacy settings as an effective means of managing the audiences for their disclosures. For example, a college student who wants to post pictures from a weekend party could block family members from seeing any content related to the event. An alternative interpretation of the low level of agreement with our disclosure measure is that users are employing the lowest common denominator strategy (see Hogan 2010), in that they choose not to make disclosures that are unsuitable for any of their audiences. This merits further research, especially when considering that those who use advanced privacy settings and have more Friends on the site report more bonding and bridging social capital. 


\subsubsection{Qualitative Study of Adult Facebook Users}

During late 2009 and early 2010, we conducted 18 in-depth interviews with adult Facebook users aged 25-55 regarding their use of the site. Among the themes to emerge, comments on privacy reflected a balancing of tensions, whereby several users commented on their attempts to maximize benefits (i.e., gains in social capital) while minimizing risks through strategies related to privacy settings, disclosures, and Friending behaviors.

Our participants exhibited a wide range of attitudes regarding the relationship between privacy and disclosures made through Facebook. On one extreme, some participants said that because they employed privacy settings to restrict access to content, they freely shared content through the site. For example, a male participant said that because he limited his profile to friends only, "there's not much I won't post in there." At the other extreme, one user's privacy concerns were so high that she rarely made disclosures of any kind through the site. When asked if she thought her decision to not actively participate in the site made it less useful for her when compared with other users, she agreed, saying, "I don't get as much out of Facebook as I think a lot of people that I know do."

Participants voiced a number of strategies for making disclosures through Facebook while managing multiple audiences. For example, a female participant said she did not post many status updates because she saw them as "polluting" her Friends' pages with irrelevant information, which might have a negative impact on people with whom she regularly interacted offline. A male graduate student described Friend Lists so he could post updates about his teaching experiences but make them non-viewable to specific groups, such as his former students or current professors, saying, "Whenever I do post, people are kind of separated into the limited profile, like the student group, and that kind of filters out what I would say to those people anyway."

An older female participant's comments most closely reflect users' attempts to maximize rewards while minimizing risks of disclosure. She said she uses Facebook because of its convenience in keeping in touch with her children, extended family, and geographically dispersed friends, but she refrains from going into depth in the content she posts to the site:

\footnotetext{
It's very public and I'm a private sort of person. So while some people would say [by] just being on Facebook, I'm sharing more about myself than they would consider reasonable or safe or whatever, I have limits to what I would post and, you know, things I won't, so it just depends. There is a balance that you can be involved in a social networking site and share personal information, but without going overboard... I have my own level of privacy concerns and I don't put a lot of things out there that other people seem to feel the need to share with the world.
}

A final theme to emerge from our interviews that relates to our variables of interest reflects the notion of "Facebook literacy" among older users, such that users who may not be familiar with the various privacy settings available to control content distribution may experience more negative outcomes of their use or may 
use the site in ways that do not promote social capital benefits. For instance, a number of the adults we spoke to commented that they were unsure about their privacy settings or did not know how to limit content to specific Friends or groups of Friends. We speculate that users with low Facebook literacy might be reluctant to engage in certain kinds of interaction on the site because they are unsure how to limit their audience, which, in turn, could lower the social capital benefits they gain from those interactions. Alternatively, if this lack of understanding leads to assumptions of privacy in a public or semi-public space, there could be negative consequences for the discloser. For example, a male participant said he had become more careful in posting content to Friends' pages after he got in trouble at work because a Friend of a Friend saw a wall post he wrote that included negative comments about a coworker. Based on these preliminary data from our interviews with adult Facebook users, we suspect that efforts to increase user awareness about our three privacy-related behaviors (especially those surrounding privacy settings) are important for enabling those with low Facebook literacy to reap social capital benefits from these tools.

\subsection{Conclusion}

In this chapter, we have argued for a conception of privacy that acknowledges that users have many options for controlling access - privacy settings are just one. Users may also choose to limit their actual disclosures by reducing the number of disclosures or limiting the content of their disclosures to mundane topics. Friending criteria also play a role. For instance, very selective Friending is one strategy by which users may control audiences. These three areas - Friending, disclosures, and privacy settings - can be seen as operating in conjunction with one another. We were not able to fully flesh out the relationships among these behaviors given our current data, but hope that future investigations will utilize more granular measures of social, technical, and communication-based activities to describe privacy strategies. Research should also explore the interactions of these behaviors among various populations. For example, two chapters in this book consider how adolescents (see Peter and Valkenburg, this volume, Chap. 16) and seniors (see Maaß, this volume, Chap. 17) navigate privacy and disclosures in an online space. These populations, often neglected in academic studies, are migrating to SNSs at a rapid rate, and their concerns and behaviors should be considered as well when developing models of privacy online.

In addition to focusing on user actions, considering the structural aspects of these technologies themselves in relation to privacy is also important. For example, it can be difficult for users to determine who can see which posting (e.g., to know who is included in the "Friend of a Friend" group), which Friends are being displayed in the News Feed, or what a privacy action (e.g., "blocking" another user on Facebook) will actually do. When on Facebook, for instance, it is fairly easy to gain access to the photo album of a non-Friend after a mutual Friend comments on a 
photo. While access to this kind of information may be positively related to bridging social capital, which is associated with novel information from weak ties, it may also result in negative personal or professional outcomes associated with the unanticipated disclosure of information about the self to unintended audiences. Helping users to understand how they can control their information by using tools in the system, and aiding in understanding the implications of those tools, allows users to choose how much they share and with whom. This kind of knowledge, and the self-efficacy that accompanies it, will help enable users to maximize the potential social capital benefits from these sites while minimizing the harms that can accompany sharing some kinds of disclosures with some audiences. As noted in our qualitative findings, the role self-efficacy plays in encouraging social capital accrual through disclosures on SNSs must be considered. boyd and Hargittai (2010) found that those with low overall Internet skills are less likely to change their Facebook privacy settings and are less confident in doing so. If these populations experience negative outcomes from their SNS use (due to less optimal use of privacy settings) and fewer positive outcomes (because they are not empowered to share disclosures that may be necessary to read these benefits), they may be less likely to continue using these sites than those with higher levels of Internet skills.

In conclusion, we believe privacy behaviors on SNSs are not limited to privacy settings; Friending behaviors and disclosures are also strategies by which users may control their audience. The degree to which users employ these strategies may be instrumental for gaining social capital and avoiding privacy risks because they give users the opportunity to calibrate their disclosures to various subsets within their overall Facebook network. The intersection of privacy and social capital is an important topic, and we are hopeful that research continues to explore this topic to help enable more equable access to "the benefits of Facebook Friends" (Ellison et al. 2007).

\section{References}

Acquisti A, Gross R (2006) Imagined communities: awareness, information sharing, and privacy on the facebook. In: Privacy enhancing technologies: 6th international workshop, PET 2006, Springer, Cambridge, pp 36-58

Adler P, Kwon S (2002) Social capital: prospects for a new concept. Acad Manag Rev 27(1):17-40 Barnes S (2006) A privacy paradox: social networking in United States. First Monday 11(9): n.p

Berger CR, Calabrese RJ (1975) Some explorations in initial interaction and beyond: toward a developmental theory of interpersonal communication. Hum Commun Res 1:99-112

Bourdieu P (2001) The forms of capital. In: Granovetter M, Swedberg R (eds) The sociology of economic life, 2nd edn. Westview Press, Boulder, pp 96-111

boyd d (2008a) Facebook's privacy trainwreck: exposure, invasion, and social convergence. Convergence 14:13-20

boyd d (2008b) Taken out of context: American teen sociality in networked publics. PhD Dissertation, University of California, Berkeley 
boyd dm, Ellison NB (2008) Social network sites: definition, history, and scholarship. J Comput Mediat Commun 13:210-230

boyd d, Hargittai E (2010) Facebook privacy settings: who cares? First Monday 15(8)

Burke M, Marlow C, Lento T (2010) Social network activity and social well-being. In: Proceedings of ACM CHI 2010: conference on human factors in computing systems, ACM, New York, pp 1909-1912

Clark HH, Brennan SE (eds) (1991) Grounding in communication. APA Press, Washington, DC

Coleman JS (1988) Social capital and the creation of human capital. Am J Sociol 94(Supplement): S95-S120

Debatin B, Lovejoy JP, Horn A, Hughes BN (2009) Facebook and online privacy: attitudes, behaviors, and unintended consequences. J Comput Mediat Commun 15:83-108

DiMicco JM, Millen DR (2007) Identity management: multiple presentations of self in facebook. In: Proceedings of the 2007 ACM conference on supporting group work, ACM Press, Sanibel Island, pp 383-386

DiMicco JM, Geyer W, Dugan C, Brownholtz B, Millen DR (2009) People sensemaking and relationship building on an enterprise social networking site. In: Proceedings of the 42 nd Hawaii international conference on system sciences. (CD-ROM), Computer Society Press, Hawaii

Donath JS, boyd d (2004) Public displays of connection. BT Technol J 22(4):71-82

Dwyer C, Hiltz SR, and Passerini K (2007) Trust and privacy concern within social networking sites: a comparison of facebook and MySpace. In: Proceedings of the Americas conference on information systems 2007, AIS, Keystone

Ellison N, Steinfield C, Lampe C (2007) The benefits of Facebook "friends": exploring the relationship between college students' use of online social networks and social capital. J Comput Mediat Commun 12:1143-1168

Ellison N, Lampe C, Steinfield C, Vitak J (2010) With a little help from my friends: how social network sites affect social capital processes. In: Papacharissi Z (ed) The networked self: identity, community, and culture on social network sites. Routledge, New York, pp 124-145

Ellison NB, Steinfield C, Lampe C (2011) Connection strategies: social capital implications of facebook-enabled communication practices. New Media Soc

Granovetter MS (1973) The strength of weak ties. Am J Sociol 78(1360):1480

Gross R, Acquisti A (2005) Information revelation and privacy in online social networks. In: Proceedings of the workshop on privacy in the electronic society, ACM, Alexandria, pp 71-80

Hampton K, Wellman B (2003) Neighboring in Netville: how the Internet supports community and social capital in a wired suburb. City Commun 2(4):277-311

Hogan B (2010) The presentation of self in the age of social media: distinguishing performances and exhibitions online. B Sci Technol Soc 30:377-386

Kim J, Lee JE (2011) The facebook paths to happiness: effects of the number of facebook friends and self-presentation on subjective well-being. CyberPsychol Behav Soc Netw 14:359-364

Ko H, Kuo F (2009) Can blogging enhance subjective well-being through self-disclosure? CyberPsychol Behav 12:75-79

Krasnova H, Spiekermann S, Koroleva K, Hildebrand T (2010) Online social networks: why we disclose. J Inf Technol 25:109-125

Kraut R, Patterson M, Lundmark V, Kiesler S, Mukopadhyay T, Scherlis W (1998) Internet paradox. a social technology that reduces social involvement and psychological well-being? Am Psychol 53:1017-1031

Lampe C, Ellison N,Steinfield C (2007) A familiar face(book): Profile elements as signals in an online social network. In: Proceedings of the SIGCHI conference on human factors in computing systems, ACM, New York, pp 435-444

Lenhart A, Madden M (2007) Social networking websites and teens: an overview. Pew Internet \& American Life Project, Washington, DC 
Lin N (2001) Building a network theory of social capital. In: Lin N, Cook K, Burt R (eds) Social capital theory and research. Transaction Publishers, New Brunswick, pp 3-30

Marwick AE, boyd d (2011) I tweet honestly, I tweet passionately: twitter users, context collapse, and the imagined audience. New Media Soc 13:113-114

Mazer JP, Murphy RE, Simonds CJ (2009) The effects of teacher self-disclosure via facebook on teacher credibility. Learn Media Technol 34:175-183

Nie NH (2001) Sociability, interpersonal relations, and the internet. Am Behav Sci 45:420-435

Olson GM, Olson JS (2000) Distance matters. Hum Comput Interact 15:139-178

Putam R (2000) Bowling Alone: The collapse and revival of American community. New York: Simon \& Schoster

Portes A (1998) Social capital: its origins and applications in modern sociology. Annu Rev Sociol 22:1-24

Quan-Haase A, Wellman B (2004) How does the internet affect social capital? In: Huysman M, Wulf V (eds) Social capital and information technology. MIT Press, Cambridge, MA, pp 113-135

Resnick P (2001) Beyond bowling together: socio-technical capital. In: Carroll J (ed) HCI in the new millennium. Addison-Wesley, New York, pp 647-672

Rheingold H (1993) The virtual community: homesteading on the electronic frontier. MIT Press, Cambridge, MA

Steinfield C, Ellison NB, Lampe C (2008) Social capital, self-esteem, and use of online social network sites: a longitudinal analysis. J Appl Dev Psychol 29:434-445

Steinfield C, DiMicco JM, Ellison NB, Lampe C (2009) Bowling online: social networking and social capital within the organization. In: Proceedings of the fourth international conference on communities and technologies, ACM, New York, pp 245-254

Strater K, Richter H (2007) Examining privacy and disclosure in a social networking community. In: Proceedings of the 3rd symposium on usable privacy and security 2007, ACM, New York, pp 157-158

Stutzman F, Kramer-Duffield J (2010) Friends only: examining a privacy-enhancing behavior in facebook. In: Proceedings of the 28th international conference on human factors in computing systems, ACM, New York, pp 1553-1562

Stutzman F, Capra R, Thompson J (2011) Factors mediating disclosure in social network sites. Comput Hum Behav 27:590-598

Tufekci Z (2008) Can you see me now? Audience and disclosure regulation in online social network sites. Bull Sci Technol Stud 11:544-564

Uslaner EM (2000) Social capital and the Net. Commun ACM 43(12):60-64

Valenzuela S, Park N, Kee K (2009) Is there social capital in a social network site?: facebook use and college students' life satisfaction, trust, and participation. J Comput Mediat Commun 14:875-901

Wellman B, Gulia M (1999) Net surfers don't ride alone: virtual communities as communities. In: Kollock P, Smith M (eds) Communities and cyberspace. Routledge, New York, pp 167-194 Westin AF (1967) Privacy and freedom. Atheneum, New York

Williams D (2006) On and off the Net: Scales for social capital in an online era. J Comput Mediat Commun 11:593-628 